\title{
Strong Interaction Dynamics from Spontaneous Symmetry Breaking of Scale Invariance
}

\author{
E.I. Guendelman* \\ Physics Department, Ben-Gurion University of the Negev, Beer-Sheva 84105, Israel
}

\begin{abstract}
Using the mechanism of spontaneous symmetry breaking of scale invariance obtained from the dynamics of maximal rank field strengths, it is possible to spontaneously generate confining behavior. Introducing a dilaton field, the study of non trivial confining and de-confining transitions appears possible. This is manifest in two ways at least: One can consider bags which contain an unconfined phase in the internal region and a confined phase outside and also one obtains a simple model for deconfinement at high Temperature from the finite temperature dynamics of the dilaton field.
\end{abstract}

\section{INTRODUCTION}

Strong interaction dynamics involves many remarkable phenomena: first, QCD at very short distances has the asymptotically free property [1]. In contrast to this, in the infrared region, we are should obtain the confined phase, where only color singlets survive, since no free quarks or gluons have been observed so far. Also at high temperatures, we expect a deconfinement phase transition [2].

Although the asymptotically free property is clearly understood theoretically, the confinement is not. Lattice gauge theory provides nevertheless numerical evidence for confinement in the context of QCD [3].

Given the theoretical difficulties concerning the description of the confining phase, several phenomenological approaches have been developed, for example the Cornell potential for heavy quarks [4] and the MIT bag model [5], which gives a comprehensive description of hadron spectroscopy.

In Ref. [6] I proposed a model where the spontaneous symmetry breaking (ssb) of scale invariance induces an effective dynamics which gives rise to confinement. Here the ssb of scale invariance originates from the dynamics of maximal rank gauge fields. The resulting theory that results in fact satisfies the requirements studied by 't Hooft [7] for perturbative confinement .

More explicit computations in the context of this model [8] have shown that it gives rise to the Cornell confining potential for static sources[4]. This model can be used also to study the interplay between confinement effects and ssb of gauge symmetry [9] .

In this paper we will see that a simple generalization of [ $[6]$ through the introduction of a dilaton field allows to enlarge the set of phenomena described. In addition to confinement we can also obtain bag structures where a deconfined phase is obtained in a central region, while confinement is obtained outside. Also, a simple description of the deconfinement phase transition at high temperature is obtained.

\section{CONFINEMENT FROM SPONTANEOUS SYMMETRY BREAKING OF SCALE INVARIANCE}

In this section, we discuss the connection between the scale symmetry breaking and confinement, introduced in Ref.[6]. For this purpose we restrict our attention to the action

$$
S_{Y M}=\int d^{4} x\left(-\frac{1}{4} F_{\mu \nu}^{a} F^{a \mu \nu}\right)
$$

where $F_{\mu \nu}^{a}=\partial_{\mu} A_{\nu}^{a}-\partial_{\nu} A_{\mu}^{a}+e f^{a b c} A_{\mu}^{b} A_{\nu}^{c}$, where $e$ is the gauge coupling constant. This theory is invariant under the scale symmetry

$$
A_{\mu}^{a}(x) \mapsto A_{\mu}^{a \prime}(x)=\lambda^{-1} A_{\mu}^{a}(\lambda x),
$$

where $\lambda$ is a constant.

*Electronic address: guendel@bgu.ac.il 
Let us introduce the following action, which is at this point equivalent to the action above

$$
\mathcal{S}_{1}\left[\omega, A_{\mu}^{a}\right]=\int d^{4} x\left[-\frac{1}{4} \omega^{2}+\frac{1}{2} \omega \sqrt{-F_{\mu \nu}^{a} F^{a \mu \nu}}\right]
$$

where, $\omega$ is an auxiliary scalar field, its scaling transformation is

$$
\omega \mapsto \lambda^{-2} \omega(\lambda x)
$$

Upon solving the equation of motion associated to the variation with respect to $\omega$, we obtain that $\omega=\sqrt{-F_{\mu \nu}^{a} F^{a \mu \nu}}$ and inserting back into (3), we obtain once again (1).

Let us now declare that $\omega$ is not a fundamental field, but that instead

$$
\omega=\epsilon^{\lambda \mu \nu \rho} \partial_{[\lambda} A_{\mu \nu \rho]}
$$

Now the variation with respect to $A_{\mu \nu \rho}$ gives rise to the equation:

$$
\epsilon^{\lambda \mu \nu \rho} \partial_{\lambda}\left[\omega-\sqrt{-F_{\mu \nu}^{a} F^{a \mu \nu}}\right]=0
$$

which is satisfied by

$$
\omega=\sqrt{-F_{\mu \nu}^{a} F^{a \mu \nu}}+M
$$

Here $M$ is a space time constant which produces the ssb of scale invariance and it is in fact associated with the spontaneous generation of confining behavior. This can be seen by considering the equations of motion in the case $M \neq 0$

$$
\nabla_{\mu}\left[\left(\sqrt{-F_{\alpha \beta}^{a} F^{a \alpha \beta}}+M\right) \frac{F^{a \mu \nu}}{\sqrt{-F_{\alpha \beta}^{b} F^{b \alpha \beta}}}\right]=0 .
$$

Then, assuming spherical symmetry and time independence, we obtain that, in addition to a Coulomb like piece, a linear term proportional to $M$ is obtained for $A^{a 0}$. A quantum computation also confirms this result [8].

Furthermore, these equations are consistent with the 't Hooft criteria for perturbative confinement. In fact in the infrared region the above equation implies that

$$
F^{a \mu \nu}=-M \frac{F^{a \mu \nu}}{\sqrt{-F_{\alpha \beta}^{b} F^{b \alpha \beta}}}
$$

plus negligible terms in the infrared sector. Interestingly enough, for a static source, this automatically implies that the chromoelectric field has a fixed amplitude. Confinement is obvious then, since in the presence of two external oppositely charged sources, by symmetry arguments, one can see that such a constant amplitude chromoelectric field must be in the direction of the line joining the two charges. The potential that gives rise to this kind of field configuration is of course a linear potential. Notice that the above equation implies that $M<0$, otherwise the electric field would be antiparallel to itself. The string tension between static quarks of opposite charge is proportional to the absolute value of $M$.

A configuration with a net charge is seen from the above equation to produce, at very large distances from the source, an electric field which is radial but constant in strength. This will in fact produce an infrared divergent total energy for the system. In general any non color singlet will be un-physical because these systems will have infinite energy. Furthermore such a configuration with net charge will detect and be attracted to any opposite charge no matter how far this will be by a linear potential, so a system with net charge can never be isolated.

\section{GENERALIZED MODELS ALLOWING BAG STRUCTURES AND DECONFINEMENT PHASE TRANSITIONS}

We now consider a model that generalizes that of the previous section and which includes also a scalar field $\phi$

$$
\mathcal{S}_{G}\left[\delta, \phi, A_{\mu}^{a}, \omega\right]=\int d^{4} x\left[-\frac{1}{4} \delta^{2}+\frac{1}{2} \delta \sqrt{-F_{\mu \nu}^{a} F^{a \mu \nu}}\right]+\int d^{4} x\left[\phi^{2}(\omega-\delta)+\frac{1}{2} \partial_{\mu} \phi \partial^{\mu} \phi-V(\phi)-\frac{1}{2} \omega^{2}\right]
$$


Now $\delta$ is a an independent field and if we were to consider just the first integral in (10), this would be the ordinary Yang Mills theory (after solving $\delta$ and inserting back into the action). The second integral represents then the departure from the usual Yang Mills theory. Here $\omega$ is not an independent field, but is again defined in terms of the three index potential

$$
\omega=\epsilon^{\lambda \mu \nu \rho} \partial_{[\lambda} A_{\mu \nu \rho]}
$$

In order to have scale invariance the potential must be quartic, that is

$$
V(\phi)=g \phi^{4}
$$

In addition to the scale transformation (2) for the gauge field, $\delta, \phi$ and $A_{\mu \nu \rho}$ have the scaling transformations

$$
\begin{aligned}
& \delta \mapsto \lambda^{-2} \delta(\lambda x) \\
& \phi \mapsto \lambda^{-1} \phi(\lambda x) \\
& A_{\mu \nu \rho} \mapsto \lambda^{-1} A_{\mu \nu \rho}(\lambda x)
\end{aligned}
$$

Here we have included a new dynamical degree of freedom, the scalar field $\phi$. This field, as a consequence of the ssb of scale invariance will acquire an expectation value and then confinement behavior will be a consequence of this. However, the expectation value can become zero under certain conditions, like in the central regions containing colored particles (this has some resemblance to the MIT bag model) and also due to the high temperature dynamics of the scalar field that can make the scalar field $\phi$ lose its expectation value.

Notice that in order to have a non-trivial dynamics for the scalar field $\phi$ it is necessary to consider not only a linear term in $\omega$ but also a quadratic term. It can be easily seen that the quadratic term in $\omega$ is exactly equivalent to a kinetic term for the three index field $A_{\mu \nu \rho}$. The consideration of the linear term in $\omega$ alone would force the scalar field $\phi$ to be a constant, i.e., non dynamical.

It is in fact the dynamics of $\omega$, or more precisely the dynamics of $A_{\mu \nu \rho}$, that is the one responsible for the ssb of scale invariance. Indeed the variation with respect to these fields gives rise to the equation

$$
\epsilon^{\lambda \mu \nu \rho} \partial_{\lambda}\left[\omega-\phi^{2}\right]=0
$$

This can be integrated very simply, giving rise to the solution

$$
\omega=\phi^{2}+M
$$

The constant $M$ gives rise to ssb of scale invariance now.

The equation obtained from the variation of $\delta$ leads to

$$
\delta=\sqrt{-F_{\mu \nu}^{a} F^{a \mu \nu}}-2 \phi^{2}
$$

and the equation obtained from the variation with respect to the $\phi$ field is

$$
\square \phi+4 g \phi^{3}-2 \phi[\omega-\delta]=0
$$

Using eqs. (17) and (18) in (19), we obtain

$$
\square \phi+4 g \phi^{3}-2\left[3 \phi^{2}-\sqrt{-F_{\mu \nu}^{a} F^{a \mu \nu}}\right] \phi-2 M \phi=0
$$

The equation of motion for the gauge field is now

$$
\nabla_{\mu}\left[\left(\sqrt{-F_{\alpha \beta}^{a} F^{a \alpha \beta}}-\phi^{2}\right) \frac{F^{a \mu \nu}}{\sqrt{-F_{\alpha \beta}^{b} F^{b \alpha \beta}}}\right]=0 .
$$

If $\phi$ is almost a constant (and non vanishing), we have the infrared confining solution (following the same arguments of the previous section) satisfying

$$
F^{a \mu \nu}=\phi^{2} \frac{F^{a \mu \nu}}{\sqrt{-F_{\alpha \beta}^{b} F^{b \alpha \beta}}} .
$$


Following the arguments of the previous section, this means that the string tension is proportional to $\phi^{2}$. In this regime we have that

$$
\sqrt{-F_{\alpha \beta}^{a} F^{a \alpha \beta}}=\phi^{2}
$$

Using this in the equation for the scalar field we obtain

$$
\square \phi+4(g-1) \phi^{3}-2 M \phi=0
$$

We see that when taking into account the effects of the gauge fields, the quartic self coupling of the scalar field is $g-1$ (notice therefore that the perturbative regime is achieved when $g$ is close to 1) and the scalar field acquires now the effective potential

$$
V_{e f f}(\phi)=(g-1) \phi^{4}-M \phi^{2}
$$

We have thus obtained a consistent effective potential for the dilaton field $\phi$ after classically integrating out the gauge fields.

We see then that if $g-1>0$ and $M>0$, then the scalar field $\phi$ will acquire an expectation value determined by the minimum of $V_{\text {eff }}$

$$
<\phi>=\left(\frac{M}{2 g-2}\right)^{\frac{1}{2}}
$$

At high temperatures [10], we will expect the effective potential for $\phi$ to acquire a term proportional to $(g-1) \phi^{2} T^{2}$ and therefore for high enough temperature compared to $\left(\frac{M}{2 g-2}\right)^{\frac{1}{2}}$, the scalar field will lose its expectation value, but then also the confining property is lost.

At zero temperature if we are close to the source of the chromoelectic field, we see from equation (20) that the square root term there will become large and the leading term will be $\phi$ independent, being indeed a Coulomb piece. This has the effect of stabilizing $\phi=0$ as the true vacuum once we approach the chomoelectric source. As in the case of high $\mathrm{T}$, losing this expectation value means losing the confining piece of the gauge fields. A bag type picture is obtained then, where the perturbative behavior holds very close to the sources and $<\phi>=\left(\frac{M}{2 g-2}\right)^{\frac{1}{2}}$ holds far enough from the source. This $\langle\phi\rangle$ induces then, in the infrared region, the confining behavior.

It is interesting to note that models that control the high temperature dynamics of strongly interacting particles using a dilaton field have been studied [11], although in this case, in terms of nuclear physics degrees of freedom.

Other models which use a scalar to generate a confinement mechanism have been studied in [12]. Such models are not constrained by a symmetry principle like scale invariance. Although very interesting, such models (in contrast to our approach) achieve confinement only as the result of a fine tuning: in [12] a dielectric function $\kappa$ is introduced which is a function of the scalar field and also a potential $U$ for the scalar field is included in the action. For confinement to be realized, there must be a value of the scalar field that satisfies both that $\kappa=0$ and at the same time, the value of the scalar field that achieves this is located at a minimum of $U$ and at an extremum of $\kappa$. This of course can be achieved only through a fine tuning.

\section{CONCLUDING REMARKS AND DISCUSSION}

We have seen that in the model presented here confinement becomes associated with the non-vanishing expectation value of the field $\phi$. The expectation value of $\phi$ is proportional to the string tension between static charges. The introduction of the scalar field $\phi$ allows us to study the dynamics that can be responsible for the confinement/deconfinement phase transition, for example at finite temperature. Also when we get close to the chromoelectric sources, we expect the expectation value of the scalar field $\phi$ to become zero.

\section{Acknowledgments}

I wish to thank E. Spallucci and P. Gaete for discussions. 
[1] H.D. Politzer, Phys. Rev. Lett. 30, 1346 (1973); Phys. Rept14 129 (1974); D.J. Gross and F. Wilczek, Phys. Rev. Lett. 30, 1343 (1973); Phys. Rev. D8: 3633 (1973); Phys. Rev. D9: 980 (1974).

[2] See for example G. Baym, "Deconfined Phases of Strongly Interacting Matter" in Quark Matter Formation and heavy Ion Collisions, M. Jacob and H. Satz, World Scientific (1982); For an up to date review see J.P. Blaizot, nucl-th/0611104

[3] K. G. Wilson, Phys. Rev. D10, 2445 (1974). The literature in this subject is huge and the uses of lattice techniques very extended, for example, for an interesting recent study of the phases of gauge theories in the context of these lattice techniques see M. Grady, Contributed to 24th International Symposium on Lattice Field Theory (Lattice 2006), Tucson, Arizona, 23-28 Jul 2006. e-Print Archive: hep-lat/0610042.

[4] E. Eichten, K. Gottfried, T. Kinoshita, K. D. Lane, and T. M. Yan, Phys. Rev. D17, 3090 (1978).

[5] A. Chodos, R.L. Jaffe, K. Johnson, C.B. Thorn and V.F. Weisskopf, Phys. Rev. D9, 3471 (1974).

[6] E.I. Guendelman, Int. J. Mod. Phys.A19 3255 (2004).

[7] G. 't Hooft, Nucl. Phys. Proc. Suppl. 121, 333 (2003).

[8] P. Gaete and E. I. Guendelman, Phys. Lett. B640, 201 (2006); P. Gaete, E.I. Guendelman and E. Spallucci, hep-th/0702067, to appear in Phys. Lett. B.

[9] P. Gaete and E. I. Guendelman, Phys. Lett.B593 151 (2004).

[10] L.Dolan and R. Jackiw, Phys. Rev. D9 3320 (1974); S. Weinberg, Phys. Rev. D9 3357 (1974).

[11] G. Kalbermann, J.M. Eisenberg and B. Svetitsky, Nucl. Phys. A600: 436 (1996).

[12] See for example W. Koepf, L. Wilets and S. Pepin, Phys. Rev. C50: 614 (1994). 